\title{
Improving the Separability of a Reservoir Facilitates Learning Transfer
}

David Norton

dnorton@byu.edu

Dan A. Ventura

ventura@cs.byu.edu

Follow this and additional works at: https://scholarsarchive.byu.edu/facpub

Part of the Computer Sciences Commons

\section{Original Publication Citation}

David Norton and Dan Ventura, "Improving the Separability of a Reservoir Facilitates Learning Transfer", Proceedings of the International Joint Conference on Neural Networks, pp.2288-2293, 29 (this first appeared in Transfer Learning for Complex Tasks: Papers from the AAAI Workshop, 28).

\section{BYU ScholarsArchive Citation}

Norton, David and Ventura, Dan A., "Improving the Separability of a Reservoir Facilitates Learning Transfer" (2009). Faculty Publications. 866.

https://scholarsarchive.byu.edu/facpub/866

This Peer-Reviewed Article is brought to you for free and open access by BYU ScholarsArchive. It has been accepted for inclusion in Faculty Publications by an authorized administrator of BYU ScholarsArchive. For more information, please contact ellen_amatangelo@byu.edu. 


\title{
Improving the Separability of a Reservoir Facilitates Learning Transfer
}

\author{
David Norton and Dan Ventura
}

\begin{abstract}
We use a type of reservoir computing called the liquid state machine (LSM) to explore learning transfer. The Liquid State Machine (LSM) is a neural network model that uses a reservoir of recurrent spiking neurons as a filter for a readout function. We develop a method of training the reservoir, or liquid, that is not driven by residual error. Instead, the liquid is evaluated based on its ability to separate different classes of input into different spatial patterns of neural activity. Using this method, we train liquids on two qualitatively different types of artificial problems. Resulting liquids are shown to substantially improve performance on either problem regardless of which problem was used to train the liquid, thus demonstrating a significant level of learning transfer.
\end{abstract}

\section{INTRODUCTION}

$\mathbf{L}$ EARNING transfer refers to the idea of transferring acquired knowledge from one domain to another similar but distinctly different domain. In machine learning it often refers to the approach of using a model already trained for success on one problem to realize instantaneous performance gains on another related problem [1], [2]. Since the topic of interest in this paper focuses on transferring knowledge rather than learning it, we refer to the process as learning transfer rather than transfer learning. Instead of following the usual route of directly training a model to accurately classify instances of a problem and then applying that model to a new problem, we take a more indirect approach. We train a filter with an objective function different from performance accuracy. We then use the output of that filter as input for another model that is trained for accuracy. The goal is to produce a single filter that will be useful for a broad range of problems with minimal or no modification. Having such a filter is the basis for an area of machine learning called reservoir computing, in which the filter is referred to as a reservoir [3]. When applying reservoir computing to learning transfer, rather than employing a whole trained model to a new problem, we utilize only the generally trained reservoir.

As an analogy, the concept of reservoir computing can be compared to support vector machines (SVMs) where the reservoir is analogous to the kernel. Taking this analogy further, what we are doing with the reservoir is essentially like building a custom kernel before training a SVM, and then successfully applying that kernel to other problems with no modification.

The specific model of reservoir computing that we use is called the liquid state machine, or LSM [4], [5]. LSMs are composed of two parts: the reservoir featuring a highly

David Norton and Dan Ventura are with the Computer Science Department, Brigham Young University, Provo, Utah (email: ghotikun@hotmail.com, ventura@cs.byu.edu).

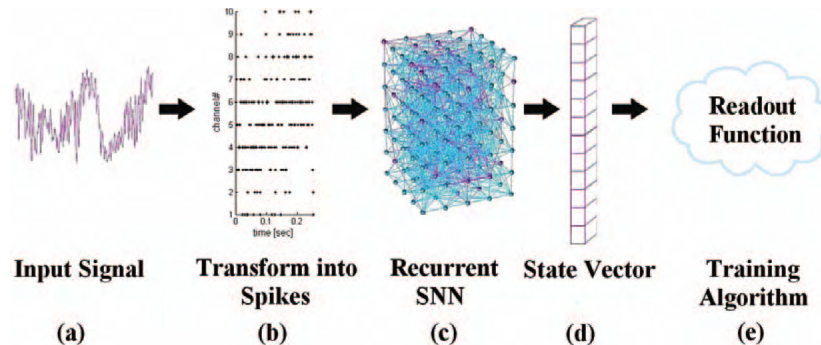

Fig. 1. Diagram of a liquid state machine. $(a, b)$ The input signal is transformed into a series of spikes via some function. (c) The spike train is introduced into the recurrent SNN, or "liquid". (d) Snapshots of the state of the "liquid" are recorded in state vectors. (e) The state vectors are used as input to train a (usually simple) learning algorithm, the readout function.

recurrent spiking neural network, and a readout function characterized by a simple learning function. Input is fed into the reservoir, or liquid, which acts as a filter. Then the state of the liquid, called the state vector, is used as input for the readout function. In essence, the readout function trains on the output of the liquid. With traditional LSMs, no training occurs within the reservoir itself. This process has been analogized with dropping objects into a container of liquid and subsequently reading the ripples created to classify the objects-hence the name liquid state machine. See Figure 1.

Because no training occurs in the reservoir, the quality of the LSM is dependent upon the ability of the liquid to effectively separate classes of input. Here the term "effectively separate" is defined as the ability of a liquid to yield unique state vectors for different classes of input, which will allow the readout function to attain acceptable accuracy. Typically, the liquid is created randomly according to some carefully selected parameters specific to the problem at hand. This parameter selection has been the topic of much research [6], [3] although the research has not yet led to a consistent and general method of generating liquids for all problems [7]. Even when adequate parameters for a given problem have been implemented, the creation of a useful liquid is not guaranteed. Typically hundreds or even thousands of liquids will be created to find a suitable filter. Fortunately, once such a liquid is discovered, the results of the LSM are comparable with the state-of-the-art [8], [3], [9]. Also, once a suitable liquid is found, it can typically be presented with other problems to good effect-the essence of learning transfer.

Since traditionally the liquids are randomly generated, the 
process of creating an effective liquid has not incorporated learning. However, we have developed a method for creating effective liquids in an LSM without having to rely on the generation of many random liquids. We do so by randomly creating a liquid in the traditional fashion and then adjusting the liquid's architecture until it can "effectively separate" as defined above. We present the liquid with sample data, observe the resulting behavior, and then use these observations to make the necessary changes to the liquid. Although this approach uses training data to modify the liquid, the objective function is based on separation rather than error. The goal is to create a liquid that will effectively separate classes of input into different patterns of state vectors. Afterwards, the readout function will learn to extract information from the state vectors via traditional error-driven learning.

In this paper we show that using this indirect approach to training a recurrent spiking neural network facilitates learning transfer. We will begin by defining a metric, called separation, that will be used to evaluate the quality of a liquid. Next we will outline our method of creating effective liquids, called Separation Driven Synaptic Modification (SDSM). Then we will provide the specific parameter settings of our experiments and describe two artificial problems we use to evaluate our algorithms. Finally we will show the results of our experiments and draw conclusions.

For duplication purposes, we note that all of the LSMs used in this paper are created using CSIM [4].

\section{SEPARATION}

Separation is a metric used to determine the effectiveness of a liquid. It essentially measures how well the liquid separates different classes of input into different reservoir states, or state vectors, and is analogous to supervised clustering. In this paper, a state vector is specifically the binary state of each neuron in the liquid at the time of the input signal's termination. Separation is calculated by dividing a set of state vectors, $O(t)$, into $N$ subsets, $O_{m}(t)$, one for each class, where $N$ is the total number of classes and $t$ is the current iteration of the liquid: defined as either a completely new random generation, or a new iteration of SDSM (explained in the next section). Individual state vectors are represented by $o$, and the more state vectors available for the metric, the more accurate the calculation of separation.

Separation is divided into two parts, the inter-class distance, $C_{d}(t)$, and the intra-class variance, $C_{v}(t) . C_{d}(t)$ is defined by Equation 1 and is the mean distance between the center of mass for every pair of classes. The center of mass for each class, $\mu\left(O_{m}(t)\right)$, is calculated with Equation 2. For clarity, we use $|\cdot|$ notation for set cardinality, and $\|\cdot\|_{k}$ notation for the $L_{k}$-norm.

$$
\begin{gathered}
C_{d}(t)=\sum_{m=1}^{N} \sum_{n=1}^{N} \frac{\left\|\mu\left(O_{m}(t)\right)-\mu\left(O_{n}(t)\right)\right\|_{2}}{N^{2}} \\
\mu\left(O_{m}(t)\right)=\frac{\sum_{o_{n} \in O_{m}(t)} o_{n}}{\left|O_{m}(t)\right|}
\end{gathered}
$$

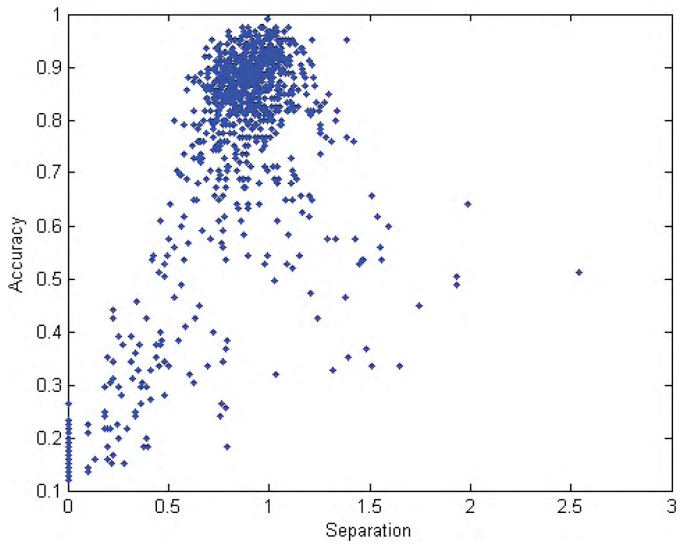

Fig. 2. Correlation between accuracy and separation in 1000 different liquids run on an artificial problem. The correlation coefficient is 0.6876 .

$C_{v}(t)$ is defined by Equation 3 and is the mean variance of every cluster, or class, of state vectors. $\rho\left(O_{m}(t)\right)$ is the average amount of variance for each state vector within class $m$ from the center of mass for that class. It is shown in Equation 4.

$$
\begin{gathered}
C_{v}(t)=\frac{1}{N} \sum_{m=1}^{N} \rho\left(O_{m}(t)\right) \\
\rho\left(O_{m}(t)\right)=\frac{\sum_{o_{n} \in O_{m}(t)}\left\|\mu\left(O_{m}(t)\right)-o_{n}\right\|_{2}}{\left|O_{m}(t)\right|}
\end{gathered}
$$

Separation can now be defined by Equation 5. $C_{v}(t)$ is incremented by one to ensure that separation never approaches $\infty$

$$
\operatorname{Sep}_{\Psi}(O(t))=\frac{C_{d}(t)}{C_{v}(t)+1}
$$

Here $\Psi$ is the liquid that produced the set of state vectors, $O(t)$. Separation essentially measures the mean distance between classes of state vectors divided by the average variance found within those classes.

In Figure 2 we show that separation does correlate with the effectiveness of a liquid. Here, effectiveness is measured as the accuracy of the LSM at classifying inputs in an artificial problem. One thousand liquids were generated with varying parameter settings to create a large variety of separation values. The artificial problem consisted of five input classes expressed as spiking patterns for four input neurons. Separation was calculated with only three examples from each class. Since we were not applying a synapse modifying algorithm to the liquids, only one iteration, $t$, was observed. The correlation coefficient between accuracy and separation is a convincing 0.6876 .

\section{iII. Separation Driven Synaptic Modification}

Separation Driven Synaptic Modification or SDSM is an approach used to modify the synapses of the liquid by using 
the separation metric defined previously and is most basically defined by the following weight update equation:

$$
w_{i j}(t+\Delta t)=\operatorname{sgn}\left(w_{i j}(t)\right)\left(\left|w_{i j}(t)\right|+E(t) \lambda F(t)\right)
$$

Here $w_{i j}(t)$ is the weight of the synapse from neuron $j$ to neuron $i$ at time $t, \lambda$ is the learning rate, $\operatorname{sgn}\left(w_{i j}(t)\right)$ is the sign of $w_{i j}(t), E(t)$ is a function of the effect of separation on the weight at time $t$, and $F(t)$ is a function of the firing behavior of all neurons in the liquid at time $t$.

First we will look at the function $E(t)$. To explain this function and its derivation it is first important to understand what we mean by relative synaptic strength, $R_{s}$, defined by Equation 7.

$$
R_{s}=\frac{\left|w_{i j}(t)\right|-\mu_{w}}{M_{w}}
$$

Here $\mu_{w}$ estimates the expected value of the magnitude of synaptic weights in the initial liquid. $M_{w}$ estimates the maximum value of random variables drawn from the same distribution used to generate synaptic weights in the initial liquid. (These approximations were obtained via simulation with 10,000 samples). $M_{w}$ essentially normalizes the synaptic strength while $\mu_{w}$ is used to differentiate weak synapses and strong synapses. A negative $R_{s}$ is considered weak while a positive $R_{s}$ is considered strong.

Recall that $C_{d}(t)$ is the mean distance between the center of mass for every pair of classes and is referred to as the interclass distance. $C_{v}(t)$ is the mean variance of each class and is referred to as the intra-class variance. Too little distance between centers of mass, $C_{d}(t)$ (Equation 1), or too much variance within classes, $C_{v}(t)$ (Equation 3), can decrease separation and thus the overall effectiveness of the liquid. Generally speaking, if there is too little distance between centers of mass, it is because strong synapses are driving the liquid to behave a particular way regardless of input class. To rectify this, we want to strengthen weak synapses and weaken strong synapses. This will drive the liquid towards a more chaotic structure that will yield results more dependent on the input. On the other hand, if there is too much variance within classes, it is because the liquid is too chaotic to allow inputs of the same class to behave similarly. To relieve this problem, it is necessary to strengthen strong synapses and weaken weak synapses even more. This will polarize the liquid, requiring greater differences in input to cause a change in the liquid's behavior (in other words, the liquid will be less chaotic).

The motivation behind the function $E(t)$ is balancing these two solutions at the level of an individual synapse. The first solution, solving the problem of differentiating classes of input, $d_{i}$, is implemented with Equation 8.

$$
\begin{aligned}
d_{i} & =\alpha_{i}\left(1-\frac{C_{d}}{S e p_{\Psi}^{*}}\right) \\
\alpha_{i} & =\frac{\sum_{k=1}^{N} \mu_{i}\left(O_{k}(t)\right)}{N}
\end{aligned}
$$

Here $\alpha_{i}$ is the activity of a specific neuron $i$ (the postsynaptic neuron of synapse $w_{i j}$ ) and is defined by Equation 9 . $\alpha_{i}$ contains $\mu\left(O_{k}(t)\right)$ which is the mean of the state vectors in class $k$. Specifically, $\mu_{i}\left(O_{k}(t)\right)$ is the value of the $i^{t h}$ element of the mean state vector. This is also the fraction of state vectors belonging to class $k$ in which neuron $i$ fires. $S e p_{\Psi}^{*}$ is the optimal separation value for the given problem and liquid. We evaluate $S e p_{\Psi}^{*}$ by approximating the maximum separation for an artificial set of $N$ state vectors that have the same cardinality as state vectors from the given liquid. The artificial set of state vectors is constructed such that for all vectors the average Hamming distance to all other vectors is (approximately) maximized.

In Equation 8, the normalized value of $C_{d}(t)$ is subtracted from one so that $d_{i}$ will provide greater correction for smaller values of $C_{d}(t)$. Essentially what Equation 8 does is to multiply the activity of a particular neuron by the amount of correction necessary for too little distance between class centers of mass. We assume that neuron activity is to blame for this problem. This may or may not be the case; however, consistently assuming correlation between $C_{d}(t)$ and neuron activity should eventually impose this correlation on the liquid and ultimately yield the desired results.

The solution to the second problem (too much variance within classes), is implemented with Equation 10.

$$
v_{i}=\frac{\sum_{k=1}^{N} \mu_{i}\left(O_{k}(t)\right) \rho\left(O_{k}(t)\right)}{N}
$$

$v_{i}$ is calculated similarly to $\alpha_{i}$ except that each instance of $\mu_{i}\left(O_{k}(t)\right)$ is multiplied by the mean variance for class $k$, because mean variance is determined class by class. The end result is that Equation 10 provides greater correction for larger values of $C_{v}(t)$ which is desirable since we are trying to reduce intra-class variance. Like the equation for $d_{i}$, the equation for $v_{i}$ assumes a correlation between the neuron's activity and $C_{v}(t)$.

The function $E(t)$ is derived from the Equations $7-10$ as follows:

$$
E(t)=R_{s}\left(v_{i}-d_{i}\right)
$$

Here $d_{i}$ is subtracted from $v_{i}$ because, as mentioned previously, we want the distance correction, $d_{i}$, to strengthen weak synapses and weaken strong synapses while we want the variance correction, $v_{i}$ to strengthen strong synapses and weaken weak synapses. In other words, we want $d_{i}$ to increase the chaotic nature of the liquid and $v_{i}$ to decrease the chaotic nature of the liquid. Ultimately the goal of Equation 11 is to find a balance between a liquid that is too chaotic and one that is too stable [10].

We now turn our attention to $F(t)$, the function of the firing behavior of all neurons in the liquid at time $t$. The function is expressed in three parts as follows:

$$
F(t)= \begin{cases}\frac{1}{\phi(t)}, & \text { if } w_{i j}(t) E(t) \geq 0 \\ \phi(t), & \text { if } w_{i j}(t) E(t)<0\end{cases}
$$




\begin{tabular}{|l|c|}
\hline Neurons & 64 \\
Connection Probability & 0.3 \\
Synaptic Weight Mean & $2 \cdot 10^{-8}$ \\
Synaptic Weight SD & $4 \cdot 10^{-8}$ \\
Samples per Class & 3 \\
Training Iterations & 500 \\
$\lambda$ & $5 \cdot 10^{-10}$ \\
Inoise & $5 \cdot 10^{-8}$ \\
$\tau$ & 0.003 \\
Synaptic Delay Mean & 0.01 \\
Synaptic Delay SD & 0.1 \\
\hline
\end{tabular}

TABLE I

PARAMETERS USED By SDSM FOR ARTIFICIAL AND PhONETIC PROBLEMS.

$$
\begin{gathered}
\phi(t)=2^{k A(t)-b} \\
A(t)=\frac{\sum_{o \in O(t)} \frac{1}{\eta}\|o\|_{1}}{|O|}
\end{gathered}
$$

Here $A(t)$ is the activity of the entire liquid at time $t$ and is calculated by finding the average fraction of neurons that fire in each state vector in $O(t)$ with $\eta$ being the total number of neurons in the liquid. $\phi(t)$ is a transformation of $A(t)$ that reduces it to a function that will allow $F(t)$ to work as a simple multiplication of $E(t)$ in Equation 6. $\phi(t)$ contains two variables, $k$ and $b$, that represent, respectively, the scale and offset of the transformation. For our experiments, $k=$ 6 and $b=3$ were found, through preliminary tuning, to yield the highest separation values. $F(t)$ uses the state of the synapse and the results of $E(t)$ to determine how the global activity of the liquid at time $t$ will effect the change in weight. The effect of $F(t)$ is to promote the overall strengthening of excitatory synapses while promoting the overall weakening of inhibitory synapses if less than half of the neurons in the liquid fire. If more than half of the neurons fire, the effect of $F(t)$ is reversed. The goal of $F(t)$ is to direct the liquid to a "useful" amount of activity. This assumes that half of the neurons firing for all state vectors is the desired fraction of activity to achieve the maximum separation possible.

\section{Parameter Settings}

We determined the best setting for each of the many parameters in the liquid by performing extensive preliminary tuning. The different parameters we looked at in these prelimnary efforts were the number of neurons, connection probability, the mean synaptic weight and delay, the standard deviation of the synaptic weight and delay, the number of samples per class used to determine separation at each instance, the number of iterations to run, the learning rate, the decay time constant, and the amount of noise present in each neuron. Table I shows the parameters we use for all of the results presented in this paper.

Some of the parameters presented in Table I require further explanation to more easily replicate the results. The connection probability is the probability that any given neuron (including input neurons) is connected to any other

\begin{tabular}{|l|cccc|}
\hline & Input 1 & Input 2 & Input 3 & Input 4 \\
\hline Class 1 & 1 & 0 & 0 & 0 \\
Class 2 & 0 & 1 & 0 & 0 \\
Class 3 & 1 & 1 & 0 & 0 \\
Class 4 & 0 & 0 & 1 & 0 \\
Class 5 & 1 & 0 & 1 & 0 \\
\hline
\end{tabular}

TABLE II

FREQUENCY PATTERNS FOR EACH CLASS IN THE FREQUENCY RECOGNITION PROBLEM. EACH INPUT REPRESENTS ONE OF FOUR INPUT NEURONS. A 1 INDICATES A FAST SPIKING FREQUENCY WHILE A 0 REPRESENTS A SLOWER SPIKING FREQUENCY.

liquid neuron (liquid neurons cannot connect back to input neurons). The value for the connection probability indicated in Table I means that each neuron is connected to roughly one third of the other neurons. The "samples per class" parameter refers to the number of training samples used from each class when calculating separation. This in turn is what drives the SDSM algorithm. The more samples used, the more accurate the separation calculation will be, but at the cost of speed. The number of iterations is simply how long to run the SDSM algorithm. In our experiments, by 500 iterations, most liquids had reached a plateau in separation improvement. $\lambda$ is the learning rate first shown in Section III. $\tau$ is the decay time constant which refers to the rate at which the membrane potential of each synapse decays. Inoise is the amount of noise produced by each neuron and is necessary for an efficient liquid [11].

\section{Artificial Problems}

Two artificial problems were developed to explore learning transfer in liquids trained with Separation Driven Synaptic Modification (SDSM). The first problem is the simpler of the two, and we call it the frequency recognition problem. This problem has four input neurons and five classes. Each input neuron fires at a slow or fast frequency, where slow neurons fire with a mean frequency of $10 \mathrm{~Hz}$ and fast neurons fire with a mean frequency of $100 \mathrm{~Hz}$. The five classes are defined by specific combinations of fast and slow input neurons as shown in Table II, where 1 represents a fast input neuron and 0 a slow one. These particular patterns were chosen to challenge the liquid with a variety of combinations as well as the task of ignoring one channel (input neuron 4). Individual samples from each class are generated by following this template and then jittering the frequencies.

The second problem is more general and complex. We call it the pattern recognition problem. This problem has eight input neurons and a variable number of classes. Each class is based on a template spike pattern randomly created for each input neuron. The random pattern is generated by plotting individual spikes with a random distance between one another. This distance is drawn from the absolute value of a normal distribution with a mean of $10 \mathrm{~ms}$ and a standard deviation of $20 \mathrm{~ms}$. Once the template pattern for each input neuron in a class is created, individual instances from the class are created by jittering each spike in the template. The spikes are jittered by an amount drawn from a zero-mean 


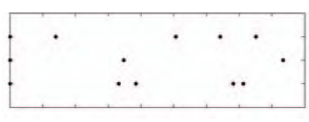

(a) Template for Class A

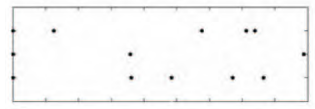

(c) Instance 1 for Class A

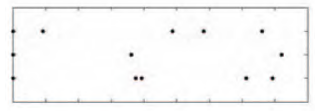

(e) Instance 2 for Class $\mathrm{A}$

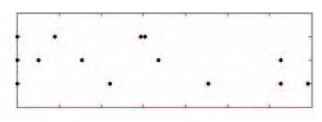

(b) Template for Class B

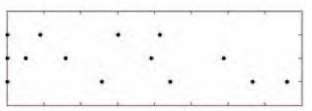

(d) Instance 1 for Class B

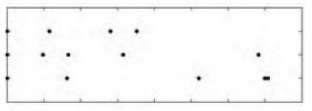

(f) Instance 2 for Class B
Fig. 3. The templates for two classes, A and B, are shown in (a) and (b) respectively. Each class has three input neurons designated by the $y$-axis. The $x$-axis is time spanning $100 \mathrm{~ms}$. (c) and (e) show examples of instances from class A created from jittered versions of the template. (d) and (f) show examples of instances from class B.

normal distribution with a standard deviation of $5 \mathrm{~ms}$ making the problem particularly difficult. A simplified example with only two classes and three input neurons is shown in Figure 3.

\section{LEARNING TRANSFER WITH SDSM}

In order to explore learning transfer, we created liquids with SDSM for both the pattern and the frequency recognition problems. For the pattern recognition problem we generated liquids for 4-, 8-, and 12-class problems. For the frequency recognition problem, we created liquids for the specifically defined 5-class scenario. We produced fifty liquids for each of these four cases via SDSM over a span of five hundred iterations. Each of the resulting two hundred liquids was then used as the reservoir for all four of the problems - three of which it was not trained on. In order to observe the effect of learning, the two hundred (untrained) randomly generated liquids used to initialize SDSM were also used as the reservoir for all four problems. This allowed us to compare traditional LSMs with LSMs generated using SDSM.

To complete the LSM and to test for accuracy, state vectors obtained from these experiments were used as input to the readout function, in this case multiple perceptrons. Each perceptron was trained to classify members of one particular class, so there were $N$ binary classifiers. The output of each perceptron was then compared, assigning the class of the perceptron with the greatest confidence to the state vector in question. This readout function was used because it is very simple, thus allowing us to focus on the quality of the liquid. For all of our experiments, the test size was one hundred samples per class.

The results of these experiments are shown in Figure 4. We refer to the problem used to create a specific liquid as the liquid source.

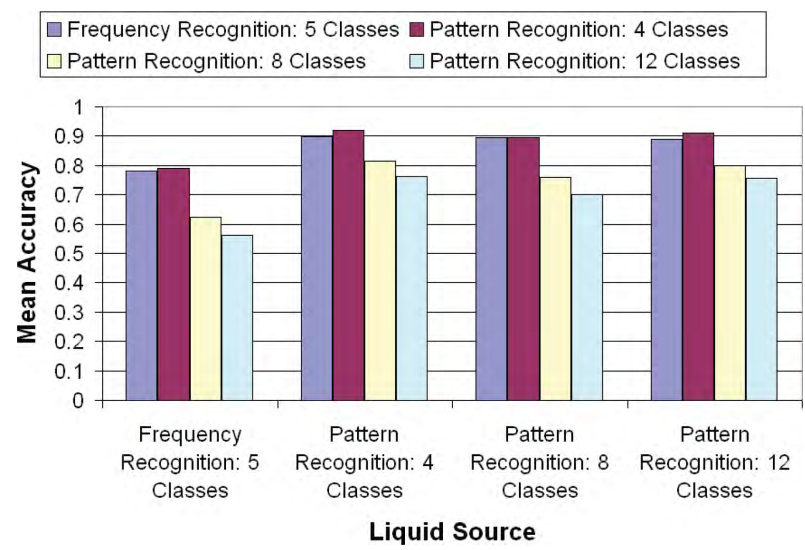

Fig. 4. The mean accuracy of LSMs using SDSM with four different liquid sources, across four different problems. The figure shows the mean accuracy of fifty unique LSMs per data point.

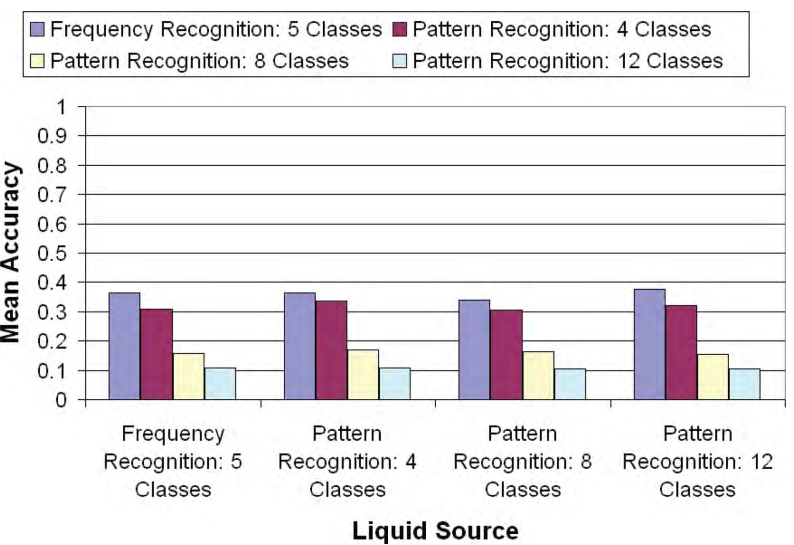

Fig. 5. The mean accuracy of LSMs using traditional (randomly generated) liquids created for the four different problems (referred to as the liquid source for convenience). The figure shows the mean accuracy of fifty unique LSMs per data point.

Input neurons are considered part of the liquid, thus their synapses are modified as part of SDSM. When a liquid is created from a source, it has $I$ input neurons, where $I$ is the number of spike trains present in the source's input. Because different problems or sources have varying numbers of spike trains, discrepancies between the number of spike trains and number of input neurons must be resolved.

When introducing a new problem to a liquid trained with a different source problem, we use the following approach. If the new problem's input is encoded in fewer spike trains than the source problem's, then the spike trains are mapped arbitrarily to a subset of the input neurons. The excess input neurons receive a null signal as input. If the new problem's input is encoded in more spike trains than the source problem's, then multiple spike trains get mapped arbitrarily to individual input neurons. The spiking patterns are combined, increasing the total number of spikes firing in each input neuron. 
The results shown in Figure 4 were obtained using two types of problems. All of the pattern recognition problems use eight spike trains for each instance while the frequency recognition problem uses only four spike trains. When a pattern recognition problem is used as the source for the frequency recognition problem, four of the input neurons have no signal. When the frequency recognition problem is used as the source for a pattern recognition problem, each input neuron combines the signals of two spike trains.

Figure 5 shows results using initial random liquids for each source. Even though the frequency recognition and pattern recognition problems have differing numbers of input neurons, the behavior of the LSMs on either problem does not change no matter which one is the source. This demonstrates the ability of LSMs to transfer a liquid between problems without dramatically effecting results of the LSM. This means that once a good filter is found, it should be usable in multiple problems. In a sense, the strengths of a filter are being transferred to another problem.

SDSM uses training data to create new liquids from those randomly generated for Figure 5 . Since the new liquids that are created depend upon the problem used to train them (the liquid source), one might expect that the transferability of the liquid would be compromised. Interestingly, the results shown in Figure 4 clearly demonstrate that this is not the case. In fact, liquids not created with frequency recognition as the source performed better on that problem than liquids actually created with frequency recognition as the source. However, liquids created with the various pattern recognition sources did perform better on those problems than liquids generated with frequency recognition as the source. In both cases, SDSM still performed significantly better than traditional LSMs (compare Figures 4 and 5). The fact that liquids created with pattern recognition performed better on both problems indicates that the source used to create the liquid can make a difference. Looking at Figure 4 we see that, on all of the problems, liquids created with pattern recognition sources outperformed liquids created with frequency recognition as the source. Pattern recognition is clearly the more complicated of the two problems; and, by applying SDSM with the more complicated source problem, the liquid may be primed for overall better performance. It should be noted that transferring learning across different numbers of classes and input neurons alone is a difficult problem that is apparently less of an issue with SDSM.

\section{CONCLUSIONS}

The nature of the liquid in an LSM allows it to transfer knowledge across a large variety of problems. Traditionally the liquid acts as a filter with the sole purpose of separating input patterns into distinct neural activation patterns. It stands to reason that a liquid capable of separating one domain of distinct input patterns might also be able to separate other domains of distinct classes. This is considered one of the strengths of LSMs. While it may take a while to find a suitable liquid, once one is found, it will likely be suitable for more than one problem. Then, for each problem a simple readout function (like a perceptron) can be effectively trained on the output of the liquid.

With SDSM, we have demonstrated a method of training the liquid to more consistently and effectively separate different input classes for a particular problem. For some problems this improvement has yielded LSMs with more than double the accuracy of traditional LSMs. Furthermore, the efficacy of learning transfer has been maintained despite the liquid being trained on a specific problem. This is especially notable since the two different types of artificial problems that we have explored have different numbers of input neurons. Learning transfer occurred despite a naïve translation from one input representation to another. Even within the pattern recognition problem type, transferring learning across problems with different numbers of classes is impressive since this essentially requires the ability to elicit a variable number of distinct responses (one for each class) within a single liquid.

The success of SDSM in allowing significant learning transfer is most likely due to the fact that the goal of the algorithm is to find a balance between chaotic and ordered behavior within the liquid architecture. The algorithm simply uses training data to evaluate the current balance within a liquid and then adjusts the liquid's structure accordingly. A large spectrum of training data could likely be used to make such an evaluation with similar results. Consequently, finalized liquids should behave similarly across a large variety of input regardless of the data used to train the liquid.

\section{REFERENCES}

[1] S. Thrun and L. Pratt, Eds., Learning to Learn. Kluwer Academic Publishers, 1998.

[2] K. Yu and V. Tresp, "Learning to learn and collaborative filtering," Neural Information Processing Systems workshop "Inductive Transfer: 10 Years Later", 2005.

[3] D. Verstraeten, B. Schrauwen, M. D'Haene, and D. Stroobandt, "An experimental unification of reservoir computing methods," Neural Networks, vol. 20, pp. 391-403, 2007.

[4] T. Natschläger, "Neural micro circuits," http:/www.lsm.turgraz.at/index.html, 2005.

[5] T. Natschläger, W. Maass, and H. Markram, "The "liquid" computer: A novel strategy for real-time computing on time series," Special Issue on Foundations of Information Processing of TELEMATIK, vol. 8, no. 1, pp. 39-43, 2002.

[6] E. Goodman and D. Ventura, "Effectively using recurrently connected spiking neural networks," Proceedings of the International Joint Conference on Neural Networks, vol. 3, pp. 1542-1547, 2005.

[7] D. Verstraeten, B. Schrauwen, and D. Stroobandt, "Adapting reservoirs to get Gaussian distributions," European Symposium on Artificial Neural Networks, pp. 495-500, 2007.

[8] E. Goodman and D. Ventura, "Spatiotemporal pattern recognition via liquid state machines," Proceedings of the International Joint Conference on Neural Networks, pp. 3848-3853, 2006.

[9] D. Verstraeten, B. Schrauwen, D. Stroobandt, and J. V. Campenhout, "Isolated word recognition with liquid state machine: a case study," Information Processing Letters, vol. 95, pp. 521-528, 2005.

[10] N. Brodu, "Quantifying the effect of learning on recurrent spiking neurons," Proceedings of the International Joint Conference on Neural Networks, pp. 512-517, 2007.

[11] K. Jim, C. L. Giles, and B. G. Horne, "An analysis of noise in recurrent neural networks: Convergence and generalization," IEEE Transactions on Neural Networks, vol. 7, pp. 1424-1438, 1996. 